\title{
Decay measurements of ${ }^{43} \mathrm{~K}\left(\beta^{-}\right)^{43} \mathrm{Ca}$ by HRS and TAS
}

\author{
A. Dhal ${ }^{a}$, G. Mukherjee ${ }^{\mathrm{b}}$, M. Bhattacharjee, V. Naik, S. Mukhopadhyay, D. Pandit, S. Pal, D. Mondal, P. Karmakar, \\ T. Roy, Md.A. Asgar, Soumik Bhattacharya, S. Bhattacharyya, C. Bhattacharya, S.R. Banerjee, and A. Chakrabarti
}

Variable Energy Cyclotron Centre (VECC), Kolkata 700064, India

\begin{abstract}
In this work, decay spectroscopy of neutron-rich ${ }^{43} \mathrm{~K}$ has been performed at the Radioactive Ion Beam (RIB) facility at Variable Energy Cyclotron Centre (VECC), Kolkata by producing it with an 18-MeV alpha beam on ${ }^{40} \mathrm{Ar}$ gas target. The beam was delivered from the K130 Cyclotron and the gas jet recoil transport system was used to move the products to a low-background site. The $\beta$-feeding intensities were measured by both high resolution $\gamma$-ray spectroscopy and total absorption $\gamma$-ray spectroscopy methods in order to address mainly the anomalies (if any) in the feeding to $\Delta \mathrm{J} \geq 1$ levels reported earlier. The decay half-life of ${ }^{43} \mathrm{~K}$ was also measured to be 22.4(1) hrs.
\end{abstract}

\section{Introduction}

$\beta$-decay measurement is one of the oldest methods for the study of nuclear structure. Apart from its importance to understand the basic nuclear physics, knowledge of the $\beta$-decay feeding intensity has importance in the applied field as well, e.g., calculation of decay head from a reactor $[1,2] . \beta$-decay is one of the very few methods by which the excited states in neutron-rich nuclei can be studied using low-intensity radioactive beams. In the present work High Resolution $\gamma$-ray Spectroscopy (HRS) and Total Absorption $\gamma$-ray Spectroscopy (TAS) of the $\beta$-decay of neutron-rich nucleus ${ }^{43} \mathrm{~K}$ have been performed to deduce the $\beta$-feeding intensities of each level in the daughter ${ }^{43} \mathrm{Ca}$. The decay of ${ }^{43} \mathrm{~K}$ was earlier studied by S.L. Waters [3] in 1972 and by E.K. Warburton et al. [4] in 1988. The decay consisted of only four excited states and ten gamma-rays. The spin and parity $\left(\mathrm{J}^{\pi}\right)$ of the ground state of ${ }^{43} \mathrm{~K}(Z=$ $19, N=24$ ) is $3 / 2^{+}$, corresponding to the odd-proton in the $d_{3 / 2}$ spherical orbital, which is known to decay to the four excited and also to the ground states in ${ }^{43} \mathrm{Ca}(Z=20$, $N=23)$. The known decay scheme of ${ }^{43} \mathrm{~K}$ [5] is shown in Fig. 1. The $3 / 2^{+}$ground state of ${ }^{43} \mathrm{~K}\left(\mathrm{~T}_{1 / 2}=22.3 \mathrm{~h}\right)$ decays mostly $(91 \%)$ to the $3 / 2^{+}$excited states in ${ }^{43} \mathrm{Ca}$ at $990 \mathrm{keV}$. However, there seems to be some anomaly in the beta feeding intensities to the rest of the levels in ${ }^{43} \mathrm{Ca}$. The $\beta$-feeding intensities to the $\Delta \mathrm{J}=1,5 / 2^{+}$and $5 / 2^{-}$ levels are $2.6 \%$ and $0.9 \%$, respectively. The difference in the intensities in these two states could be understood as the difference in parity in these two states. The higher energy state has no change in parity (compared with the decaying state in ${ }^{43} \mathrm{~K}$ ) while for the other one, the parity is changed, so the intensity is low. This is also in line with the difference in feeding intensities to the $3 / 2^{+}$and $3 / 2^{-}$ states. The ground state spin-parity of the daughter ${ }^{43} \mathrm{~K}$ is $\mathrm{J}^{\pi}=7 / 2^{-}$. Therefore, the $\beta$-decay intensity to this state, with $\Delta J=2$ with parity change, is expected to be much

\footnotetext{
a e-mail: a.dhal@vecc.gov.in

b e-mail: gopal@vecc.gov. in
}

smaller than the feeding intensity to the $\Delta J=1,5 / 2^{-}$ state (same change in parity). The ground state feeding intensity of $1.5 \%$ is, therefore, seems to be unusually high.

This large feeding can be attributed to the large overlap of the wave function between the ground states of ${ }^{43} \mathrm{~K}$ and ${ }^{43} \mathrm{Ca}$ due to structural effect. However, to confirm the nuclear structure effect, one needs to be ensured that there is no systematic error involved in the reported feeding intensities. The feeding intensities to the different levels of the daughter were obtained (by HRS method) from the difference in the intensities of the $\gamma$-rays feeding in and decaying out of the level. In these high resolution measurements, there are two main difficulties: low detection efficiency and the fragmentation of gamma intensity. The photopeak efficiency of the HPGe detectors is low for the high-energy $\gamma$-rays. Therefore, many weak high-energy transitions may remain unobserved in the high resolution measurements and, hence, the feeding intensity would be wrongly deduced. In these cases, higher feeding intensity incorrectly assigned to low lying levels or to the ground state. This is what is termed as 'Pandemonium effect'[6]. The solution to this experimental problem is to adopt a different approach to the measurement using a TAS which is sensitive to the $\beta$ population of the nuclear levels instead of individual $\gamma$ rays. In this work we embark our investigation to address this issue.

\section{Experimental details}

Radioactive ${ }^{43} \mathrm{~K}\left(\mathrm{~T}_{1 / 2}=22.3 \mathrm{hrs}\right)$ have been produced at the RIB facility [7] of Variable Energy Cyclotron Centre (VECC), Kolkata, India using the reaction ${ }^{40} \mathrm{Ar}\left({ }^{4} \mathrm{He}, 1 \mathrm{p}\right){ }^{43} \mathrm{~K}$ at $18 \mathrm{MeV}$. The target was a gas cell of $50 \mu \mathrm{m}$ thick ' $\mathrm{Ta}$ ' window filled with ${ }^{40} \mathrm{Ar}$ at $1 \mathrm{~atm}$. of pressure placed in the RIB beam line inside the vault area. The primary alpha $\left({ }^{4} \mathrm{He}\right)$ beam (up to $1 \mu \mathrm{A}$ of beam current) of energy $28 \mathrm{MeV}$, from the K130 cyclotron at VECC, was degraded by about $10 \mathrm{MeV}$ within the gas cell window. The reaction products, along with ${ }^{43} \mathrm{~K}$, trapped in

(c) The Authors, published by EDP Sciences. This is an Open Access article distributed under the terms of the Creative Commons Attribution License 4.0 (http://creativecommons.org/licenses/by/4.0/). 


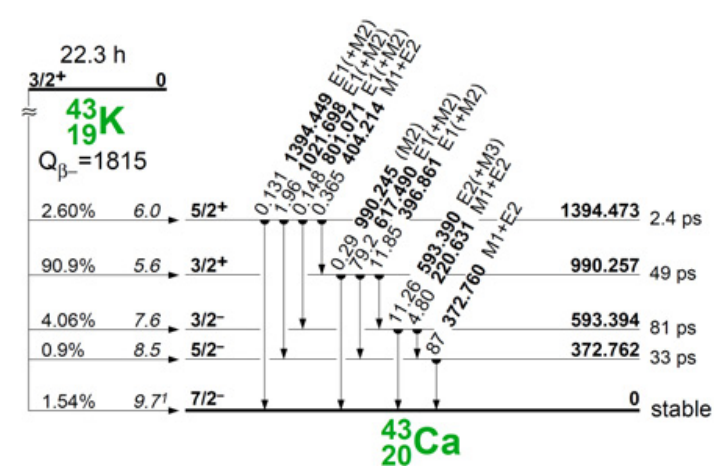

Figure 1. Decay scheme of ${ }^{43} \mathrm{~K}[5]$.

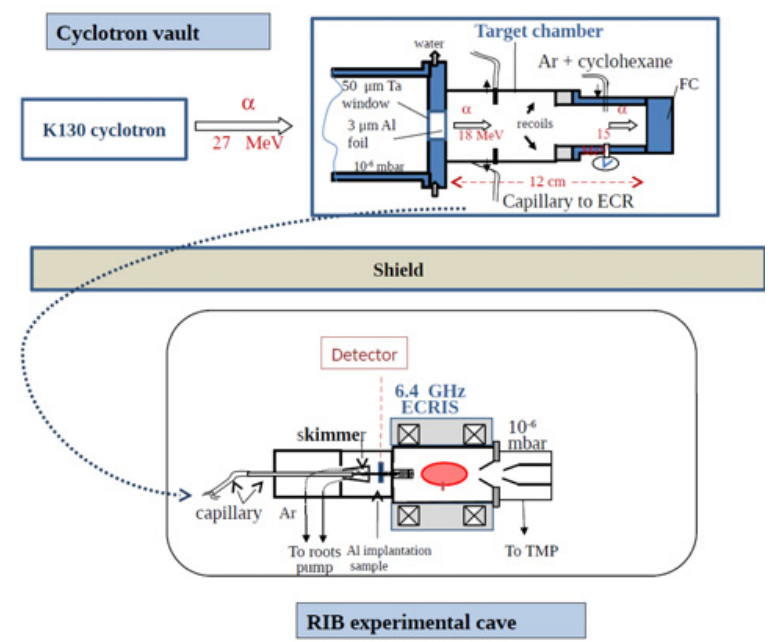

Figure 2. Schematic diagram of ${ }^{43} \mathrm{~K}$ RIB production and transportation to low background area for implantation.

organic aerosols were transferred from the target chamber to a low background site by Gas Jet Recoil Transport (GJRT) technique [8] and collected on the surface of an 'Al' catcher foil, placed after a skimmer and before a $6.4 \mathrm{GHz}$ ECR ion source. A schematic of the ${ }^{43} \mathrm{~K}$ RIB production and transportation is depicted in Fig. 2. Off-line decay measurements of the 'Al' catcher foils were done in both High-Resolution $\gamma$-ray Spectroscopy (HRS) and Total Absorption $\gamma$-ray Spectroscopy (TAS) methods.

\subsection{HRS}

In HRS, a clover HPGe detector and the single-crystal HPGe detector, procured under Nuclear Data Physics Centre of India (NDPCI), were used in singles and coincidence modes. The 'Al' catcher foil was placed at a distance of $4 \mathrm{~cm}$ from each of the detectors front faces. The dimension of the single-crystal HPGe detector is $60 \mathrm{~mm} \times 53 \mathrm{~mm}$, with a $300 \mu \mathrm{m}$ Be window. Relative efficiency is $\sim 29 \%$ for $1332 \mathrm{keV}$ of ${ }^{60} \mathrm{Co}$ source. The dimension of the clover HPGe detector is $4 \times(60 \mathrm{~mm} \times$ $53 \mathrm{~mm}$ ). Relative efficiency, in addback mode, is $\sim 130 \%$ for $1332 \mathrm{keV}$ of ${ }^{60} \mathrm{Co}$ source. Energy resolutions for the single-crystal and clover HPGe detectors are $2.28 \mathrm{keV}$ and $2.54 \mathrm{keV}$ (addback) respectively, at $1332 \mathrm{keV}$ of ${ }^{60} \mathrm{Co}$. For the measurement of decay half-life, data on fixed time interval of $1 \mathrm{hr}$. each were also collected in the singles mode. The HRS set-up consisting of the clover and singlecrystal HPGe detector is shown in Fig. 3.

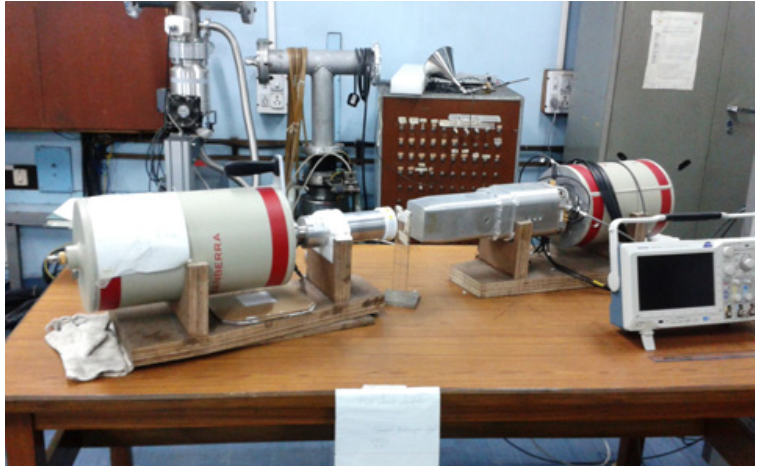

Figure 3. The HRS set-up, consists of one single crystal and one clover HPGe detectors.

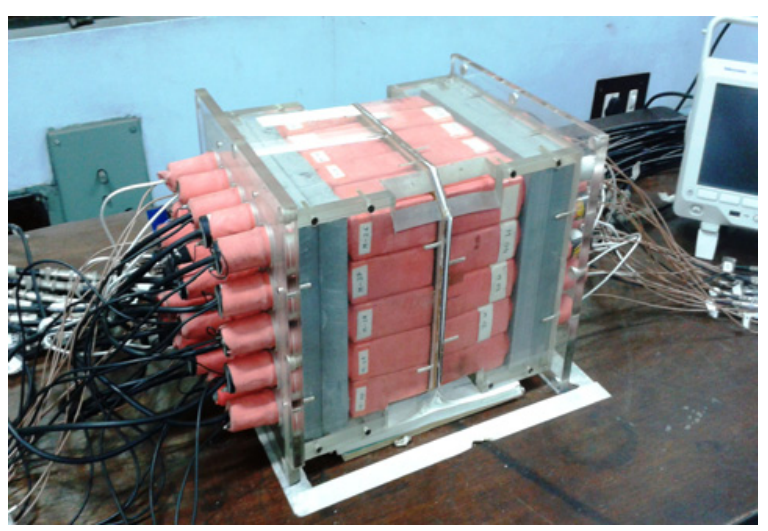

Figure 4. The TAGS set-up at VECC, in closed configuration.

\subsection{TAS}

For the TAS measurement the modular TAGS facility $[9,10]$ at VECC with 50 element BaF2 detectors in a $4 \pi$ geometry with capabilities of different fold selections has been used. 'Al' catcher foil was placed at the centre of the $\mathrm{BaF} 2$ array. The length of each detector is $5 \mathrm{~cm}$ with a cross sectional area of $3.5 \times 3.5 \mathrm{~cm}^{2}$. The energy resolution for an individual detector was about $8 \%$ at $1.17 \mathrm{MeV}$ of ${ }^{60} \mathrm{Co}$. The time resolution is $\sim 450 \mathrm{ps}$ between any two detectors. The efficiency of the array is $\sim 86 \%$ for $662 \mathrm{keV}$ of ${ }^{137} \mathrm{Cs}$.

Other details about the detectors and their characterization can be found in $[10,11]$. The full array (shown in Fig. 4) consists of two blocks having 25 detectors in each one of them. The detectors in each block are arranged in castle type geometry. The blocks are brought together for a closed $4 \pi$ configuration. The radiation source can be kept inside and at the middle of the array. The large granularity of $\mathrm{BaF} 2$ detectors make the present set-up unique. The advantages of the large granularity is to separate the sum peaks from the individual peaks to get a clean sum spectrum. This can be achieved in the offline analysis by incorporating the condition of multiplicity hit in obtaining the sum energy, in the modular spectrometer. Energy calibration and gain matching of the crystals were done before obtaining the sum spectrum. A multiplicity spectrum, defined by the number of crystals hit in an event, was generated and the sum spectra were obtained using different condition of multiplicity (M). Sum peaks can be easily identified from the individual ones to get a clean sum peak spectrum. Moreover, one can identify the number of 


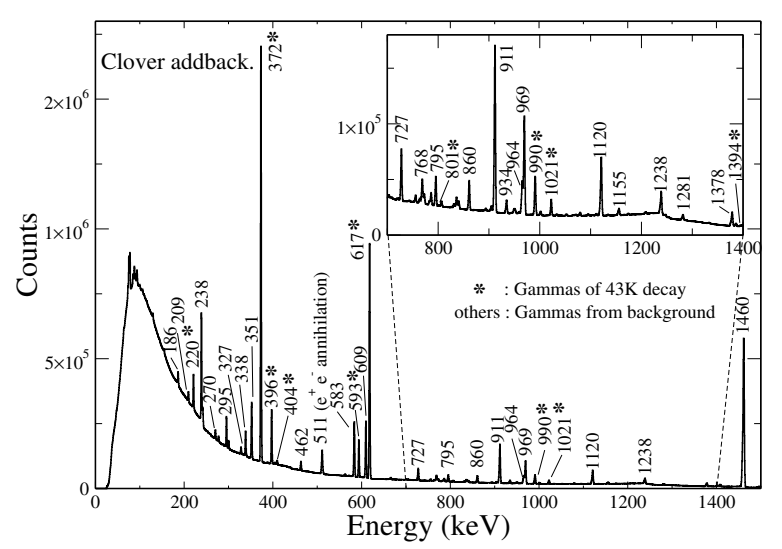

Figure 5. Gamma spectrum shows the $\gamma$-lines from ${ }^{43} \mathrm{~K}$ decay (star marked) and room background.

individual $\gamma$-rays contributing to a particular sum peak. The details about the procedure can be found in [9].

\section{Results and discussions}

A representative $\gamma$-ray spectrum from the clover addback is shown in Fig. 5. This spectrum shows only the ${ }^{43} \mathrm{~K}$-decay lines(marked with stars) and the standard identified room background lines with no other radioactive impurities. The production and decay of different nuclei are consistent with the calculated excitation function as obtained from PACE4 for the reaction ${ }^{4} \mathrm{He}+{ }^{40} \mathrm{Ar}$ at $18 \mathrm{MeV}$ beam energy. Except ${ }^{42} \mathrm{~K},{ }^{43} \mathrm{~K}$ and ${ }^{39} \mathrm{Ar}$ nuclei, all other nuclei produced in the above mentioned reaction are stable. Population cross-section of ${ }^{42} \mathrm{~K}$ is negligible at $18 \mathrm{MeV}$ of alpha energy and ${ }^{39} \mathrm{Ar}$ decays $100 \%$ to the ground state (stable) of ${ }^{39} \mathrm{~K}$. Therefore, we have gammarays only from the decay of ${ }^{43} \mathrm{~K}$ and standard room background.

Further verification of existence of long lived activity, if any, are done by comparing three data sets (as shown in the Fig. 6) taken, for $1 \mathrm{hr}$. each, at different times after the implantation was stopped. The Fig. 6 shows decay data taken after $2 \mathrm{hr}$. 6 min., $15 \mathrm{hr} .6 \mathrm{~min}$. and $71 \mathrm{hr}$. 6 min., respectively, after the implantation stopped. The Fig. 6(b) shows that the intensity of $\gamma$-lines decaying from ${ }^{43} \mathrm{~K}$ are decreasing with time where as the intensity of background $\gamma$-lines remains constant. The Fig. 6(c) is very useful not only to determine the strength of the sample of ${ }^{43} \mathrm{~K}$ decays (from the amount collected on the foil) compared to the background radioactivity after 3 half-lives of the sample are past but also to check the existence of any longer lived contaminant in the sample. It can be seen from this spectrum that many of the $\gamma$-ray peaks from the sample are close or less than the background peaks. Therefore, at this time, the background $\gamma$-rays are dominating the spectrum.

The decay curve was plotted, as shown in Fig. 7, with each data point corresponding to $2 \mathrm{hrs}$. data. We have obtained a decay half-life of $\mathrm{T}_{1 / 2}=22.4(1) \mathrm{hrs}$ for ${ }^{43} \mathrm{~K}$ by fitting the decay curve of the most intense $372-\mathrm{keV}$ $\gamma$-ray as shown in Fig. 2. The measured half-life is in good agreement with the earlier measurement [12] for this nucleus. The weighted average value of $\mathrm{T}_{1 / 2}$ of present and three earlier results $[3,12,13]$ is $22.28(7) \mathrm{hrs}$.

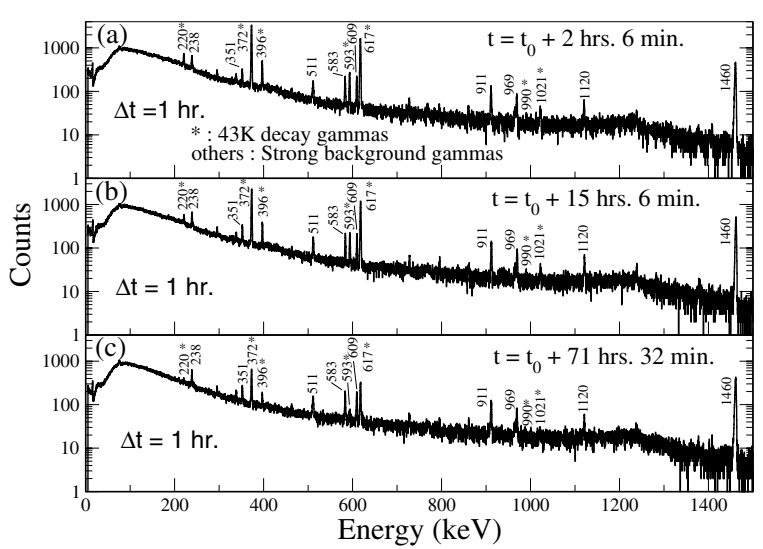

Figure 6. Gamma-ray spectra, of $1 \mathrm{hr}$. duration each, for different times after the irradiation stopped.

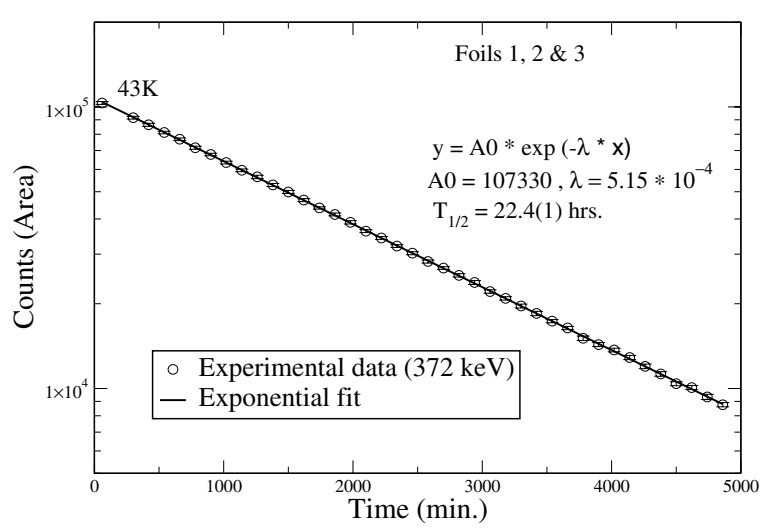

Figure 7. Decay curve for $372 \mathrm{keV}$ gamma of ${ }^{43} \mathrm{~K}$ decay.

Taking into account the detector efficiency and total conversion co-efficient, relative intensities of $\gamma$-rays and $\beta$-feeding of ${ }^{43} \mathrm{~K}$ decay for the HPGe single crystal detector is calculated. In the HRS data, this is obtained from the difference of feeding-in and feedingout intensities in each level. The preliminary results show some deviations from the earlier reported values [5]. For example, an increase of about $1.4 \%$ in $\beta$-feeding for the $3 / 2^{+}$state has been deduced in the present work and correspondingly the feeding intensity to the ground state comes out to be $\sim 0.3 \%$. In the TAGS data the sum-energy peaks correspond to the level energies and the intensities of these sum-energies directly give the feeding intensities. In this work, the sum energy spectra are obtained by putting different multiplicity fold-gates as shown in Fig. 8. In Fig. 8(a), the spectra are gated by multiplicity fold greater than a set value where as the spectra in Fig. 8(b) are obtained with a particular multiplicity fold gate. It can be seen that the peaks which result from the summing (e.g., $990 \mathrm{keV}$ and $1394 \mathrm{keV}$ ) of more than one $\gamma$-rays could easily be identified in the spectra of Fig. 8(b). The intensities of these sum-peaks will give the feeding intensities of the corresponding level.

The feeding pattern can only be obtained after applying an unfolding procedure to the measured spectra using the detector response function using the relation:

$$
\mathbf{d}=\mathbf{R}(\mathbf{B}) \times \mathbf{f}
$$




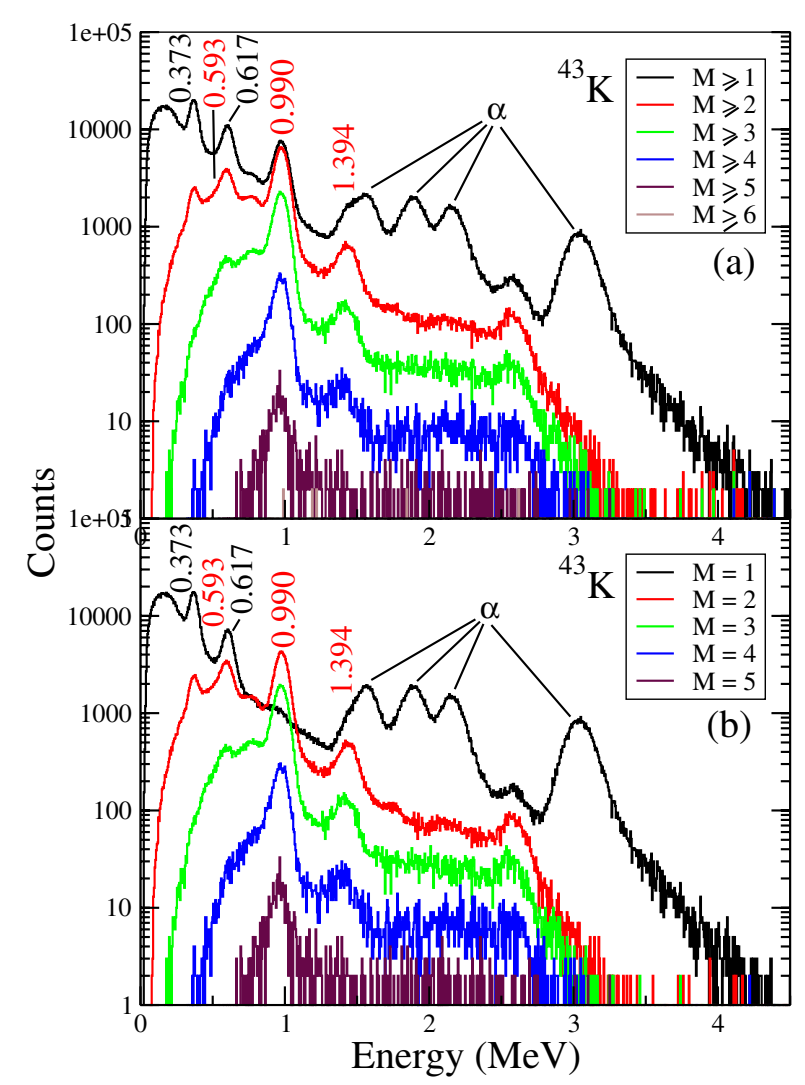

Figure 8. Sum spectra of ${ }^{43} \mathrm{~K}$ decay from the TAGS data obtained with different minimum multiplicity gate (a) and with exact multiplicity gate (b). The sum peaks (e.g., at $990 \mathrm{keV})$ are easily identified from the spectra in (b).

where $\mathbf{d}$ represents the measured data, $\mathbf{R}$ is the response matrix of the detector, which depends on the branching ratios $(\mathbf{B})$ of the levels in the daughter nucleus and $\mathbf{f}$ is the feeding distribution we wish to determine. The response function $\mathbf{R}$ can only be calculated using Monte Carlo techniques $[14,15]$ and this procedure has been used in recent works $[1,16]$ on TAS measurements on ${ }^{239} \mathrm{Pu}$ and ${ }^{92} \mathrm{Rb}$. Detailed analysis of the TAGS data of the present experiment is in progress, as per the above mentioned formalism, to obtain the feeding intensities.

\section{Summary}

The neutron rich ${ }^{43} \mathrm{~K}$ nucleus has been produced using the RIB beam line at VECC by $\alpha$-induced fusion evaporation reaction using gas target and the gas-jet system was used to move the products for offline decay spectroscopy study using high resolution and total absorption spectroscopy methods. Precise value of the decay half-life has been obtained and the preliminary analysis of the $\beta$-feeding intensity show some deviation from the reported values.

We thank the staff members of the K130 Cyclotron for providing good quality and uninterrupted $\alpha$-beam during the experiment. Help and cooperation of personnel of RIB lab., VECC are highly appreciated.

\section{References}

[1] A. Algora et al., Phys. Rev. Lett. 105, 202501 (2010)

[2] A. Algora et al., J. Korean Phys. Soc. 59, 1479s (2011)

[3] S.L. Waters, Radiochimica Acta 17, 63 (1972)

[4] E.K. Warburton et al., Phys. Rev. C38, 2822 (1988)

[5] National Nuclear Data Centre, http://www. nndc. bnl.gov/ensdf/

[6] J.C. Hardy et al., Phys Lett. B 71, 307 (1977)

[7] A. Chakrabarti, Nucl. Instr. and Meth. B 261, 1018 (2007)

[8] A. Bandyopadhyay et al., Nucl. Instr. and Meth. A 562, 41 (2006)

[9] G. Mukherjee et al., EPJ Web of Conferences 66, 11026 (2014)

[10] D. Pandit et al., Nucl. Instr. and Meth. A 624, 148 (2010)

[11] S. Mukhopadhyay et al., Nucl. Instr. and Meth. A 582, 603 (2007)

[12] J.F. Emery et al., Nucl. Sci. Eng. 48, 319 (1972)

[13] S. Hontzeas et al., Can. Jour. of Chem. 41, 2194 (1963)

[14] D. Cano-Ott et al., Nucl. Instr. and Meth. A 430, 488 (1999); 430, 333 (1999)

[15] J.L. Tain and D. Cano-Ott, Nucl. Instr. and Meth. A 571, 728 (2007); 571, 719 (2007)

[16] A.-A. Zakari-Issoufou et al., Phys. Rev. Lett. 115, 102503 (2015) 